\title{
Notch1 Signaling Regulates Radial Glia Differentiation through Multiple Transcriptional Mechanisms
}

\author{
Brooke A. Patten, ${ }^{1}$ S. Pablo Sardi, ${ }^{1}$ Samir Koirala, ${ }^{1}$ Masato Nakafuku, ${ }^{2}$ and Gabriel Corfas ${ }^{1}$ \\ ${ }^{1}$ Division of Neuroscience, Children's Hospital, Harvard Medical School, Boston, Massachusetts 02115, and 2Division of Developmental Biology, Cincinnati \\ Children's Hospital Medical Center, Cincinnati, Ohio 45229-3039
}

Signaling by the Notch1 receptor is critical for the formation of radial glia in the developing nervous system. We have shown previously that Notch1 regulates the molecular and morphological differentiation of radial glia through the transcriptional activation of at least two genes, brain lipid binding protein (BLBP) and the erbB2 receptor tyrosine kinase. However, the mechanisms by which this occurs remained undefined. Here we demonstrate that Notch1 effects on radial glia gene expression are mediated by two downstream mechanisms, one that the depends on Suppressor of Hairless $[\mathrm{Su}(\mathrm{H})]$ and the other on Deltex1 (DTX1). These two Notch1-binding proteins contribute to the regulation of BLBP and erbB2 expression, respectively. Importantly, our results suggest that, although these events can occur simultaneously, a hierarchical relationship might exist between DTX1 and $\mathrm{Su}(\mathrm{H})$, because overexpression of DTX1 or a dominantnegative form of this protein inhibits $\mathrm{Su}(\mathrm{H})$-mediated events but not vice versa. In contrast to the effects of DTX1 overexpression, interference RNA-mediated knock-down of DTX1 blocks Notch1-induced erbB2 promoter activation and radial glia formation selectively, without affecting $\mathrm{Su}(\mathrm{H})$-dependent pathways, indicating that loss of DTX1 expression and expression of dominant-negative DTX1 result in different alterations in cell differentiation and gene expression. Together, these results show that Notch1 regulates radial glia formation through two distinct transcriptional mechanisms and that the outcomes of Notch1 signaling may depend on the relative expression levels of its coregulators.

Key words: Notch1; $\mathrm{Su}(\mathrm{H})$; erbB; cerebellum; glia; BLBP; DTX

\section{Introduction}

Neuronal migration is a critical step in brain development. Neurons are generated in germinal layers located in the walls of the neural tube and then migrate to the position they will occupy in the mature brain. Newborn excitatory cortical neurons and most cerebellar neurons migrate along specialized cells called radial glia (Rakic 1971a,b). These cells extend radial processes from the germinal layers through the developing anlage and thus provide a scaffold for migrating neurons. In the cerebellum, Bergmann glia cells are the radial glia that guide and support the migration of granule neurons (Goldowitz and Hamre, 1998).

Bergmann glia have been used as a model to study the mechanisms that regulate radial glia formation and function in vivo and in vitro (Sotelo and Changeux, 1974; Hatten, 1985; Rio et al., 1997; Borghesani et al., 2002; Patten et al., 2003; Yue et al., 2005). Hatten (1985) originally demonstrated that contact with granule neurons induces cerebellar astroglia to adopt a radial glia pheno-

Received Nov. 9, 2005; revised Jan. 10, 2006; accepted Feb. 3, 2006.

This research was supported in part by a National Institute of Neurological Disorders and Stroke R01 NS35884 (G.C.), Mental Retardation Research Center/National Institutes of Health (NIH) Grant P30-HD 18655 (G.C.), a Lefler fellowship (S.P.S.), Fundamental Neurobiology NIH Training Grant T32 NS007484-06 (S.K.), and Predoctoral NIH Training Grant T-32 AG00222 (B.A.P.). We thank Jean Brennan, Pieter Dikkes, Josh Murtie, and Kristine Roy for help with aspects of this study. We thank Elior Peles for the contactin plasmid and Christiane Richter-Landsberg for the OLN-93 cells.

Correspondence should be addressed to Dr. Gabriel Corfas, Division of Neuroscience, Children's Hospital, 300 Longwood Avenue, Boston, MA 02115. E-mail: gabriel.corfas@tch.harvard.edu.

DOI:10.1523/JNEUROSCI.4829-05.2006

Copyright $\odot 2006$ Society for Neuroscience $\quad$ 0270-6474/06/263102-07\$15.00/0 type. This transformation includes the formation of radial processes (Hatten, 1985) and the expression of the radial glia marker brain lipid binding protein (BLBP) (Feng et al., 1994). More recently, we showed that this radial glia differentiation depends on activation of the Notch1 receptor and subsequent downstream transcriptional events (Patten et al., 2003). When Notch1 receptors expressed by cerebellar astroglia are activated by contact with granule neurons, which express Jagged1, Notch 1 signaling induces the transcription of BLBP and the receptor tyrosine kinase erbB2. Upregulation of BLBP is important for adhesion between migrating neurons and radial glia (Feng et al., 1994), although the increase in erbB receptor expression makes the glia responsive to the erbB ligand neuregulin1 (NRG1), which is also expressed by the granule neurons (Rio et al., 1997). NRG1-erbB receptor signaling then induces the morphological transformation of the astroglia into radial glia (Patten et al., 2003).

The studies described above also suggested that multiple intracellular pathways mediate the effects of Notch 1 signaling in radial glia. In canonical Notch signaling, ligand binding induces receptor proteolysis, releasing the Notch1 intracellular domain (NICD). NICD then translocates to the nucleus and binds to Suppressor of Hairless [ $\mathrm{Su}(\mathrm{H})$ ] [also known as C-promotor binding factor 1 (CBF1), RBP-J, and LAG1], converting it from transcriptional repressor to transcriptional activator (for review, see Lai, 2002). We found that one effect of Notch1 on radial glia formation, the activation of BLBP expression, depends on this canonical $\mathrm{Su}(\mathrm{H})$ pathway. However, we were surprised by the 
observation that the Notch1-mediated erbB2 expression and glial morphological differentiation were independent of $\mathrm{Su}(\mathrm{H})$ (Patten et al., 2003), indicating that noncanonical Notch1 signaling is involved in the formation of radial glia.

Deltex1 (DTX) has been implicated in noncanonical Notch1 signaling (Kishi et al., 2001) and was originally identified in Drosophila as a positive regulator of Notch1 function (Xu and Artavanis-Tsakonas, 1990; Matsuno et al., 1995; Ramain et al., 2001). There are four mammalian DTX proteins named DTX1 through DTX4 (Kishi et al., 2001; Storck et al., 2005). These proteins form homodimers and heterodimers with each other and bind to the ankyrin repeats of NICD (Kishi et al., 2001; Yamamoto et al., 2001; Takeyama et al., 2003). Although in mammalian cells DTX is seen mostly as a positive regulator of Notch1, it has been suggested that it could also act as a negative regulator (Matsuno et al., 1998; Sestan et al., 1999). However, the mechanisms by which DTX contributes to Notch1 signaling, its potential gene targets, and the ways in which DTX and $\mathrm{Su}(\mathrm{H})$ interact in mammalian cells remain undefined.

Here we show that DTX1 function is necessary for the Notch1-mediated regulation of radial glia differentiation, with DTX1 activity being necessary for the activation of erbB2 transcription and radial glia morphological differentiation. Furthermore, our experiments indicate that a hierarchical relationship might exist between DTX1 and $\mathrm{Su}(\mathrm{H})$, the first being dominant over the latter. These results provide new insights into mechanisms of brain development, in particular how Notch 1 signals to regulate transcription of multiple genes that can influence cell differentiation.

\section{Materials and Methods}

In situ hybridization. The DTX1 probe was generated by PCR with two primers: forward, 5' -GCAAGAGCAGCAGAGATAG-3'; and reverse, 5' GGATAGACAGAGGTCAAGAG-3'. The 514 bp PCR product was then cloned into pCRII-TOPO expression vector (Invitrogen, Carlsbad, CA) to generate the sense and antisense probes. The preparation of digoxigenin (DIG)-labeled riboprobe for DTX1 and the in situ analysis was performed as described by Messersmith et al. (2000). Briefly, FVBN wildtype mice (Charles River Laboratories, Wilmington, MA) were fixed by intracardial perfusion with 4\% paraformaldehyde in PBS, and the cerebellum was dissected, cryoprotected, and cut in $15 \mu \mathrm{m}$ parasagittal sections. DIG-labeled riboprobes were hybridized to cryosections, detected using an alkaline phosphatase-conjugated anti-DIG antibody (Roche Products, Welwyn Garden City, UK), and developed with a nitrobluetetrazolium-chloride/5-bromo-4-chlor-indolyl-phosphate substrate (Roche Products). When immunostaining and in situ analysis were combined, the in situ protocol was followed as described, and then sections were fixed in $4 \%$ paraformaldehyde for $10 \mathrm{~min}$, rinsed with PBS, blocked in $4 \%$ horse serum with $0.1 \%$ Triton X-100, and probed with a rabbit anti-calbindin antibody (1:50; Abcam, Cambridge, MA), followed by an anti-mouse HRP-conjugated secondary antibody (Jackson ImmunoResearch, West Grove, PA).

Immunostaining. Cells in culture were fixed in $4 \%$ paraformaldehyde for $10 \mathrm{~min}$, rinsed with PBS, blocked in $4 \%$ horse serum with $0.1 \%$ Triton $\mathrm{X}-100$, and probed with a rabbit anti-GFAP (1:500; DakoCytomation, High Wycombe, UK) or a mouse anti-green fluorescent protein (GFP) (1:200; Santa Cruz Biotechnology, Santa Cruz, CA) antibody, followed by anti-rabbit cyanine 3 or anti-mouse Alexa Fluor 488-conjugated secondary antibody (Jackson ImmunoResearch). Nuclei were stained with Hoechst 33342 (Invitrogen).

Glia and neuron cell culture. Primary astroglial and granule neurons were purified from postnatal day 6 (P6) Long-Evans rat or FVBN mouse cerebella (Charles River Laboratories) by selective preplating as described by Patten et al. (2003). Briefly, cerebella were excised in PBS and dissociated in astrocyte media [DMEM, 10\% fetal bovine serum (FBS), and $2 \mathrm{~mm}$ L-glutamine (Invitrogen)] with $0.02 \%$ DNase. Cells were plated onto uncoated dishes to remove fibroblasts, and then the supernatant was plated onto three $10 \mathrm{~cm}$ poly-D-lysine $(0.5 \mu \mathrm{g} / \mathrm{ml}$; BD Biosciences, Franklin Lakes, NJ) coated dishes to attach glia. Plates were washed with PBS to remove and collect neurons. Glia were passaged 3-4 $\mathrm{d}$ later to remove any remaining neurons, producing a glial culture $>95 \%$ pure. All cells were incubated at $37^{\circ} \mathrm{C}$ with $5 \% \mathrm{CO}_{2}$.

Cell lines. The oligodendrocyte cell line (OLN-93) and HEK 293T and NIH3T3 cell lines were grown in DMEM with high glucose, 10\% FBS, and $2 \mathrm{~mm}$ L-glutamine (Invitrogen).

Generation of lentiviruses for interference RNA expression. The vector pLL3.7 (from Luc Van Parijs, Massachusetts Institutes of Technology, Cambridge, MA) was used to generate small hairpin RNAs as described by Rubinson et al. (2003). The following oligonucleotides were subcloned into the HpaI/XhoI sites (nucleotides targeting DTX are underlined): interference RNA (RNAi) DTX1-A, forward, 5' - TGGGCCGAAAGGTGCTGAGATT CAAGAGATCTCAGCACCTTTCGGCCCTTTTTTC-3'; RNAi DTX1-A, reverse, $5^{\prime}$-TCGAGAAAAAAGGGCCGAAAGGTGCTGAGATCTCTTGA ATCTCAGCACCTTTCGGCCCA-3'; RNAi DTX1-B, forward, 5'-TCGG CCTACGACATGGACATTTCAAGAGAATGTCCATGTCGTAGGCC GTTTTTTC-3'; and RNAi DTX1-B, reverse, 5'-TCGAGAAAAAA CGGCCTACGACATGGACATTCTCTTGAAATGTCCATGTCGTAGGC CGA-3'.

Lentiviral production was performed as described by Rubinson et al. (2003). Briefly, HEK 293T cells were cotransfected with pLL3.7 and packaging vectors, and the resulting supernatant was collected after 48 and $72 \mathrm{~h}$. Lentiviruses were recovered after ultracentrifugation for $1.5 \mathrm{~h}$ at 25,000 rpm in a Beckman Instruments (Fullerton, CA) SW28 rotor and resuspended in PBS.

Contactin purification. Two $10 \mathrm{~cm}$ dishes of $70 \%$ confluent HEK 293T cells were transfected with a rat Ig-contactin plasmid (Peles et al., 1995). Two days later, cells were passed onto three $15 \mathrm{~cm}$ dishes and incubated with Optimem media (Invitrogen) and penicillin/streptomycin (Invitrogen) for $24 \mathrm{~h}$. Medium was collected and replaced for another $24 \mathrm{~h}$. The conditioned media was added to $1 \mathrm{ml}$ of protein $\mathrm{A} / \mathrm{G}$ beads, washed with PBS, and rotated overnight at $4^{\circ} \mathrm{C}$. The beads were then washed twice with PBS, eluted twice with $3 \mathrm{ml}$ of $0.1 \mathrm{M}$ glycine, $\mathrm{pH} 2.5$, for $15 \mathrm{~min}$ each, and eluted once with PBS for $5 \mathrm{~min}$. Eluates were neutralized with $1 \mathrm{M}$ Tris, $\mathrm{pH} 8$, and centrifuged at $3000 \times g$ for $5 \mathrm{~min}$ in a $30 \mathrm{kDa}$ Centrifugal Filtration Device (Amicon, Beverly, MA).

Plasmids. The 1.7 kb BLBP promoter (Feng and Heintz, 1995), the 4.5 kb erbB2 promoter (White and Hung, 1992), and the CBF1-dependent reporter (Hsieh et al., 1996) were subcloned into a pGL3basic luciferase reporter vector (Promega, Madison, WI) as described by Patten et al. (2003). The wild-type $\mathrm{Su}(\mathrm{H})$ construct encodes a full-length Xenopus $\mathrm{Su}(\mathrm{H})$, and the dominant-negative $(\mathrm{DN})-\mathrm{Su}(\mathrm{H})$ construct encodes a Xenopus $\mathrm{Su}(\mathrm{H})$ with mutated DNA binding domains (Wettstein et al., 1997). The NICD construct encodes the entire intracellular domain of the Notch1 receptor [formerly referred to as FCDN1 by Patten et al. (2003)] (Shawber et al., 1996). The wild-type DTX1 construct encodes the full-length human Deltex (amino acids 1-620), and the DN-DTX1 construct encodes a truncated human Deltex (amino acids 1-412) (Yamamoto et al., 2001). To generate the flag-tagged mouse DTX1 construct (flag-DTX1), mouse DTX1 (BC053055, clone 8170690; American Type Culture Collection, Manassas, VA) was cloned into the NotI/XbaI sites of pA1-fg using the following primers: 5'-GAAGGCGGCC GCCATCAG TTCCGGCAAGACACAG-3'; and 5'-CGATTCTAGA TCAGGCCTTGGCCATGGCCTC-3'.

Transfections. Primary astrocytes at $70 \%$ confluence were transfected with Fugene 6 reagent (Boehringer Mannheim, Indianapolis, IN), according to the recommended protocol. To detect the transfected cells, cells were cotransfected with a plasmid encoding GFP at a molar ratio of 3:1 (target plasmid/GFP plasmid). Previously, the level of coexpression of the target genes and GFP was found to be $>95 \%$, as described by Patten et al. (2003).

Luciferase assay. Cells were transfected and, $2 \mathrm{~d}$ later, lysed and assayed using the Dual Assay Luciferase kit (Promega), following the recommended protocol. Cotransfected TK-renilla luciferase was used to normalize samples for transfection efficiency and sample handling. If cells infected with RNAi viruses were used for the assay, they were infected $10 \mathrm{~d}$ before transfection. 
More than $98 \%$ of the cells were infected as determined by GFP expression. The results presented represent the mean \pm SEM of at least three independent experiments.

Astroglial morphology assay. Glia were plated at 10,000 cells $/ 200 \mathrm{~mm}^{2}$. The next day, cells were transfected, and, $24 \mathrm{~h}$ later, freshly dissociated neurons were added at a ratio of 10:1 (neurons/glia). When glia infected with RNAi viruses were used, they were infected $10 \mathrm{~d}$ before transfection. More than $98 \%$ of the cells were infected as determined by GFP expression. The radial glia morphology was determined as described by Patten et al. (2003). Briefly, cells were fixed with $4 \%$ paraformaldehyde and labeled with a rabbit anti-GFAP antibody (DakoCytomation) and the appropriate secondary antibody. The morphology of cells in random fields was assessed in cells that expressed both GFAP and GFP, as determined by immunostaining. Glia were considered to be radial when they had at least one thin process longer than 50 $\mu \mathrm{m}$. This criterion was based on previous studies (Hatten, 1985; Rio et al., 1997). The results presented represent the mean \pm SEM of at least three independent experiments, and 200-400 cells were examined for each assay.

Western blot. To test for RNAi knock-down of DTX1, HEK 293T cells were cotransfected with plasmids encoding flag-DTX1 and control, DTX1-A, or DTX1-B RNAi constructs in a 1:6 molar ratio. After $24 \mathrm{~h}$, samples were then lysed in SDS sample buffer, separated by SDSPAGE on a $12 \%$ gel, and transferred to polyvinylidene difluoride (PVDF) membranes (Millipore, Bedford, MA). Blots were probed with a mouse anti-flag antibody (Sigma, St. Louis, $\mathrm{MO})$, stripped, and then probed with a mouse anti-glyceraldehyde-3-phosphate dehydrogenase (GAPDH) antibody (Chemicon, Temecula, CA) for loading control. To test for the effectiveness of contactin, OLN-93 cells were lysed in SDS sample buffer, separated by SDSPAGE on a $4-20 \%$ gradient gel (Invitrogen), and transferred to PVDF membranes (Millipore). Blots were probed with a mouse anti-MAG antibody (Chemicon), stripped, and then probed with a rabbit anti-GAPDH antibody (Chemicon). Detection was performed using the appropriate HRP-conjugated secondary antibodies (Jackson ImmunoResearch) and developed using Western Lightning chemiluminescence reagent (PerkinElmer, Wellesley, MA) with autoradiography film (Denville Scientific, Metuchen, NJ).

Reverse transcription-PCR. Total RNA was extracted from the cerebellum of FVBN P6 wild-type mice by first homogenizing the tissue with a Polytron homogenizer (probe PT-DA 1205/2EC; Kinematica, Cincinnati, $\mathrm{OH}$ ) and then extracting using Trizol reagent (Sigma) according to the recommended protocol. Total RNA was extracted from cultured cells using RNeasy spin columns (Qiagen, Valencia, CA) with DNase treatment both on column (Qiagen) and off column (RNase-free DNase I kit; Invitrogen), according to the recommended protocol.

Statistical analysis. Statistical significance for the astroglial morphological assays was determined by $\chi^{2}$ test using the Statview program. Statistical significance for the luciferase assays was determined by Wilcoxon's signed-rank test.

\section{Results}

\section{DTX1 expression is developmentally regulated in} the cerebellum

To determine whether DTX1 might play a role in Bergmann radial glia during cerebellar development, we first analyzed its

\section{DTX1}

C

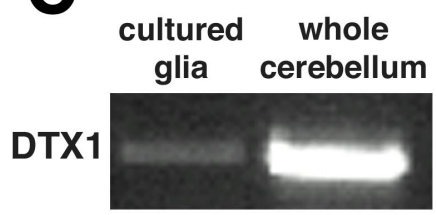

D

cultured glia

NIH3T3

DTX1

$-$

Figure 1. DTX1 is expressed by Bergmann glia in the developing cerebellum and cultured astrocytes. $\boldsymbol{A}$, Parasagittal sections (to P6 (top) and adult (bottom) cerebellum were analyzed by ISH for DTX1 mRNA expression. At P6, DTX1 signal is to DTX1.D, Purified cerebellar glia and NIH3T3 cells were lysed and subjected to Western blot analysis with an anti-DTX1 antibody that detected a band at $70 \mathrm{kDa}$. EGL, External granule cell layer.

pattern of expression by in situ hybridization (ISH). At P6, a time when the primary role of Bergmann glia is to support the migration of cerebellar granule cells, strong DTX1 expression could be detected in cells of the Purkinje cell layer, in which Bergmann glia cell and Purkinje neuronal cell bodies are located. Similar results were obtained at P0 (data not shown). The cell bodies of the DTX1-expressing cells were small, suggesting that they belong to the Bergmann glia rather than the large Purkinje cell bodies. To confirm this interpretation, we combined DTX1 ISH with immunostaining for calbindin, a marker of Purkinje cells, which showed that the DTX1 ISH signal was not in these neurons (Fig. $1 B$ ). Interestingly, although DTX1 expression in Bergmann glia was high during early postnatal development, it became undetectable in the adult, after neuronal migration is complete (Fig. $1 A$ ).

The expression of both Notch1 and DTX1 by Bergmann glia in the developing cerebellum (Irvin et al., 2001; Patten et al., 2003) suggested that Notch1-DTX1 signaling might play important roles in the formation and/or function of radial glia. To test this, we turned to cocultures of P6 cerebellar granule neurons and cerebellar astroglia, a well established in vitro assay (Hatten, 1985; Mason et al., 1988; Rio et al., 1997) that we used previously to study the roles of Notch1 signaling in radial glia formation (Pat- 
ten et al., 2003). Importantly, reverse transcription (RT)-PCR (Fig. 1C) and Western blot analysis (Fig. 1D) showed that cerebellar astroglia in culture express DTX1, indicating that the cocultures could be a useful experimental system to test the roles of this protein.

\section{Neurons regulate radial glia morphological differentiation through Notch1-DTX1 signaling}

Using this in vitro assay, we showed previously that some aspects of the Notch1-induced differentiation of cerebellar astroglia into radial glia, i.e., the acquisition of radial morphology, occur through a pathway independent of $\mathrm{Su}(\mathrm{H})$ (Patten et al., 2003). To begin to determine whether DTX1 is critical for these $\mathrm{Su}(\mathrm{H})$ independent events, we made use of a dominant-negative form of DTX1 (DN-DTX1). This truncated DTX1, which lacks the C-terminus domain III, can bind to NICD and has been shown to block Notch1-DTX signaling (Yamamoto et al., 2001). We first tested whether DN-DTX1 expression inhibits the ability of neurons to induce the morphological differentiation of radial glia. Expression of DN-DTX1, but not wild-type DTX1, blocked neuronal-induced morphological differentiation (Fig. $2 A, B$ ), indicating that DTX function is necessary for this event. To examine whether the effects of DN-DTX1 reflect alterations in Notch signaling, we tested whether DN-DTX1 blocks the glial morphological change induced by expression of the constitutively active NICD. Cerebellar astroglia were transfected with plasmids encoding NICD, DTX1, and DN-DTX1 alone or in combination. Similar to what we observed in neuron-glia cocultures, expression of DN-DTX1 blocked the ability of NICD to induce radial glia formation (Fig. 2C,D). Interestingly, DN-DTX1 expression also lowered the number of radial glia in unstimulated cultures (Fig. 2C).

\section{Neurons mediate erbB2 expression via \\ Notch1-DTX1 signaling}

Previously, we showed that the induction of radial glia morphological differentiation mediated by neuronal contact and by Notch 1 signaling depends on the transcriptional activation of the erbB2 gene, also a $\mathrm{Su}(\mathrm{H})$-independent process (Patten et al., 2003). Therefore, we tested whether the induction of erbB2 transcription by Notch1 signaling and neuronal contact is mediated by DTX. An erbB2-luciferase reporter construct (Patten et al., 2003) was cotransfected into cerebellar astrocytes with plasmids encoding NICD, DN-DTX1, DTX1, or a control plasmid. NICD expression activated the erbB2 promoter, as we showed previously (Patten et al., 2003), and this effect was blocked by DNDTX1 (Fig. 2E). Similar to what we observed for glial morphology (Fig. 2C), expression of DN-DTX1 reduced the basal level of erbB2 promoter activity (Fig. $2 E$ ). In contrast, expression of wild-type DTX1 alone had no effect on basal erbB2 activity and did not affect the ability of NICD to regulate transcription. Consistent with these observations, expression of DN-DTX1 also blocked the induction of erbB2 transcription by neuronal contact (Fig. 2F). Together, these results suggest that Notch1-DTX1mediated erbB2 transcription is part of the mechanism by which neurons regulate radial glia morphological differentiation.

\section{Expression levels of $\mathrm{Su}(\mathrm{H})$ and DTX1 determine Notch1 downstream transcriptional events}

The results described above indicate that Notch1 signaling regulates radial glia differentiation through two different pathways. On one hand, Notch1-Su(H) signaling mediates the acquisition of radial glia identity, defined by the expression of the radial glia
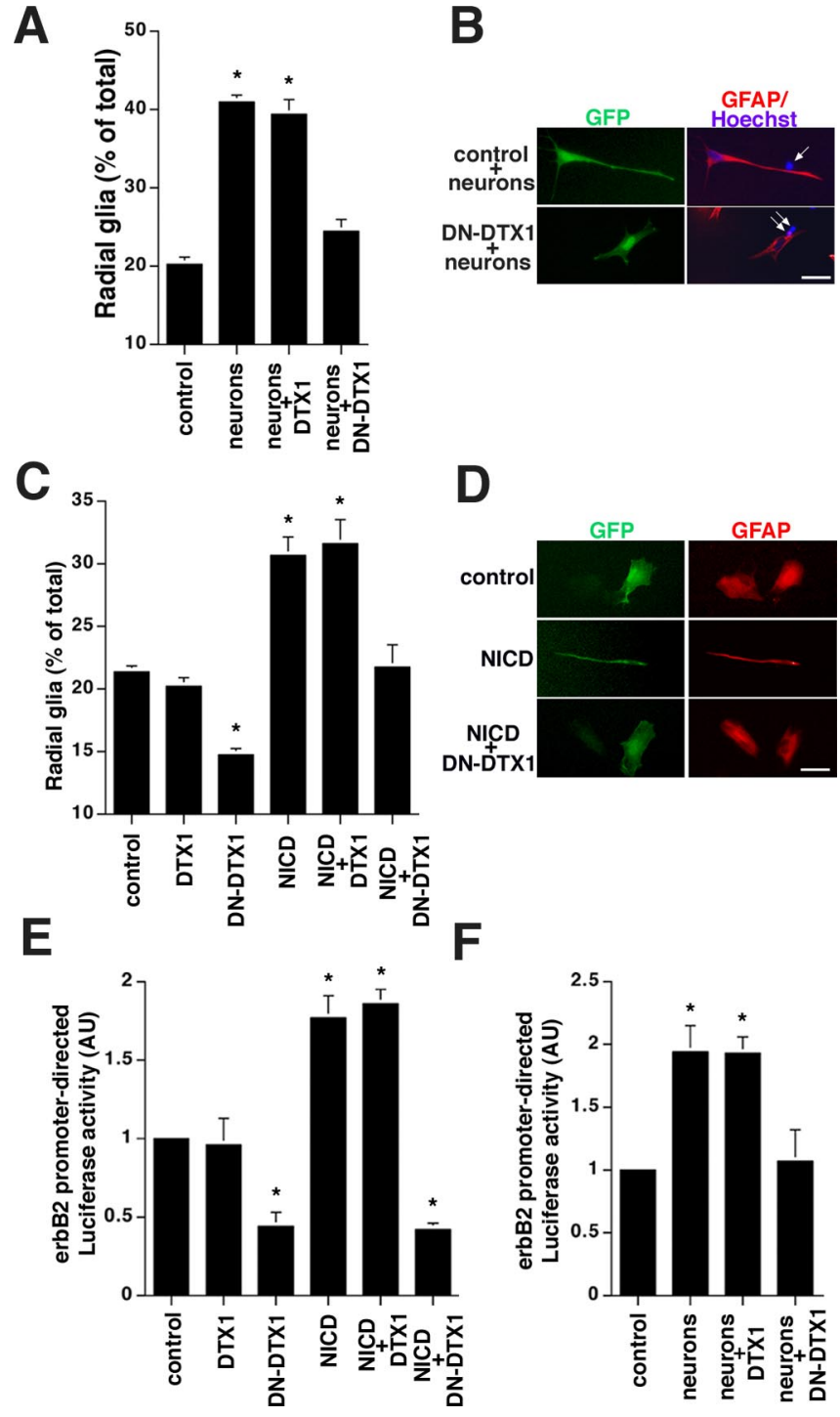

Figure 2. DTX1 mediates Notch1-induced radial glia morphological differentiation and erbB2 expression. A, Purified P6 cerebellar glia were transfected with a plasmid encoding DTX1, DN-DTX1, or a control plasmid. Twenty-four hours later, purified P6 granule neurons were added, and the next day glia morphology was examined. ${ }^{*} p<0.007$, conditions that are significantly different from control. $\boldsymbol{B}$, Images show representative glia treated with neurons and transfected with a plasmid encoding DN-DTX1 or a control plasmid. Glia were stained with antibodies against GFP (green) or GFAP (red), and nuclei were stained with Hoechst (blue). Arrows indicate neuronal nuclei. Scale bar, $25 \mu \mathrm{m}$. C, Purified P6 cerebellar glia were transfected with a plasmid encoding DTX1, DN-DTX1, a control plasmid, and/or NICD. Twenty-four hours later, glial morphology was examined. Radial glia were identified as GFAP $(+)$ cells with at least one thin process longer than $50 \mu \mathrm{m} .{ }^{*} p<0.03$, conditions that are significantly different from control. $\boldsymbol{D}$, Images show representative glia under control conditions or transfected with a control plasmid or plasmids encoding NICD and/or DN-DTX1. Glia were stained with antibodies against GFP (green) and GFAP (red). Scale bar, $25 \mu \mathrm{m}$. E, Purified P6 cerebellar glia were cotransfected with plasmids encoding the erbB2-Luciferase reporter and DTX1, DNDTX1, NICD, or a control plasmid. Twenty-four hours later, the cells were lysed and luciferase activity was measured. ${ }^{*} p<0.03$, conditions that are significantly different from control. $\boldsymbol{F}$, Purified P6 cerebellar glia were transfected with plasmids encoding DTX1, DN-DTX1, or a control plasmid. Twenty-four hours later, neurons were added to the cultured, and the next day cells were lysed and luciferase activity was measured. ${ }^{*} p<0.03$, conditions that are significantly different from control. AU, Arbitrary units.

gene BLBP (Patten et al., 2003; Anthony et al., 2005). On the other hand, Notch1-DTX1 signaling contributes to the morphological differentiation of radial glia, which depends on the transcriptional activation of the erbB2 gene. Because $\mathrm{Su}(\mathrm{H})$ and 
DTX1 bind to overlapping regions of NICD (Wettstein et al., 1997; Yamamoto et al., 2001) and it has been reported that overexpression of DTX1 can prevent the binding of $\mathrm{Su}(\mathrm{H})$ to NICD (Yamamoto et al., 2001), it was important to explore whether the $\mathrm{Su}(\mathrm{H})$ - and DTX-dependent pathways interact during the process of radial glia formation. To this end, we tested whether overexpression of one of these Notch1 mediators would alter signaling through the other in cerebellar astroglia.

To study the effects of DTX1 on $\mathrm{Su}(\mathrm{H})$ dependent signaling we tested whether expression of DTX1 or DN-DTX1 alters the NICD-induced activation of a CBF1 and a BLBP reporter construct. The CBF1 reporter contains four tandem arrayed CBF1 binding sites upstream of the firefly luciferase gene (Hsieh et al., 1996) and is commonly used to test Notch1-Su(H) signaling. The BLBP reporter contains the $1.7 \mathrm{~kb}$ BLBP promoter (Feng and Heintz, 1995), which we and others have shown to be regulated by Notch1-Su(H) signaling (Patten et al., 2003; Anthony et al., 2005). To study the effects of $\mathrm{Su}(\mathrm{H})$ on DTX-dependent signaling, we tested whether expression of $\mathrm{Su}(\mathrm{H})$ alters the induction of erbB2 reporter activation and radial glia morphology by NICD or neuronal contact. Surprisingly, expression of either DTX1 or DN-DTX1 reduced the ability of NICD to induce $\mathrm{CBF} 1$ reporter activity (Fig. $3 A$ ) and completely eliminated the NICDinduced BLBP reporter activity (Fig. $3 B$ ). In contrast, $\mathrm{Su}(\mathrm{H})$ did not alter the DTXmediated events, i.e., the activation of erbB2 transcription (Fig. 3C) and the morphological differentiation of the glia (Fig. $3 D)$. Similar results were obtained previously with $\mathrm{DN}-\mathrm{Su}(\mathrm{H})$ (Patten et al., 2003). These results suggest that there is hierarchical relationship between DTX1 and $\mathrm{Su}(\mathrm{H})$ and indicate that the endogenous expression levels of DTX1 and $\mathrm{Su}(\mathrm{H})$ could be critical for determining the biological outcome of Notch1 activation during radial glia differentiation.

\section{DTX1 knock-down by RNAi prevents radial glia} morphological differentiation and induction of erbB2 expression without altering $\mathrm{Su}(\mathrm{H})$-dependent events The observation that DN-DTX1 not only blocks DTX-dependent events but also $\mathrm{Su}(\mathrm{H})$-dependent ones indicated that the interpretation of experiments using DN-DTX1 could be confounded by the promiscuity of DN-DTX1. Thus, to test the roles of DTX1 in radial glia differentiation and transcription specifically, we used RNAi knock-down of DTX1. Two lentiviruses expressing different RNAi constructs targeted toward mouse DTX1; DTX1-A and DTX1-B were generated. The ability of these RNAi constructs to knock down DTX1 expression was proven measuring the levels of an epitope-tagged DTX1 (Fig. 4A). Similarly, DTX1 RNAi also knocked down expression of endogenous DTX1 in NIH3T3 cells (Fig. 4 B). We then tested whether DTX1 knockdown inhibits the ability of neurons to induce radial glia differ-
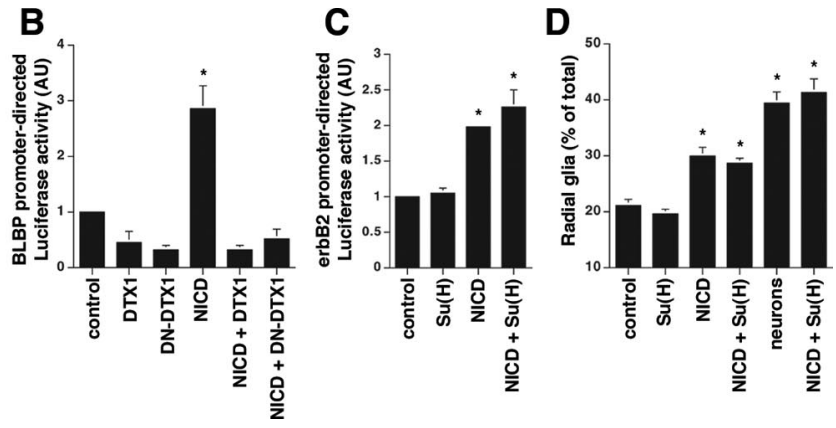

Figure 3. DTX1 overexpression inhibits Su(H)-dependent Notch1 signaling. $\boldsymbol{A}, \boldsymbol{B}$, Purified P6 cerebellar glia were transfected u(H), NICD, or a control plasmid. Twenty-four hours later, the cells were lysed and luciferase activity was measured.

A

Figure 4. RNAi knock-down of DTX1 in glia eliminates DTX-dependent events without altering Su(H)-mediated ones. $\boldsymbol{A}$, HEK 作 measured. ${ }^{*} p<0.005$, conditions that are significantly different from control. AU, Arbitrary units.

entiation. Murine astroglia were infected with a DTX1-A, DTX1-B, or a control lentivirus, and the effects of neuronal contact on radial morphology were examined. DTX1 knock-down abolished the ability of neurons to induce radial glia morphology (Fig. 4C). Similarly, DTX1 knock-down also abolished the NICD induction of erbB2 expression (Fig. 4D). In contrast, DTX1 knock-down had no effect on the ability of NICD to regulate the transcription of the CBF1 and BLBP reporters (Fig. $4 E, F$ ), indicating that $\mathrm{Su}(\mathrm{H})$ activity was normal in the absence of DTX1 expression. Together, these results further demonstrated the critical role of DTX1 in regulating erbB2 expression and radial glia morphological differentiation.

\section{Discussion}

Our experiments show that Notch 1 signaling controls the formation of radial glia through at least two distinct intracellular pathways, each regulating the transcription of different genes necessary for radial glia function, BLBP and an erbB receptor. Transcriptional activation of these genes requires the Notch1 coregulators $\mathrm{Su}(\mathrm{H})$ and DTX1, respectively. Importantly, we found that the biological outcomes of knocking down DTX1 by RNAi and expressing DN-DTX1 are different. Whereas knock-down of DTX1 blocks DTX-mediated events without affecting $\mathrm{Su}(\mathrm{H})$ sig- 
naling, overexpression of DN-DTX1 blocks both DTX- and $\mathrm{Su}(\mathrm{H})$-mediated events. This apparent nonspecific effect of DNDTX1, which likely reflects a competition between DN-DTX and $\mathrm{Su}(\mathrm{H})$ for binding to NICD (Yamamoto et al., 2001), indicates that some studies in which the biological roles of DTX-mediated Notch1 signaling were studied using DN-DTX1 could now be interpreted differently. Interestingly, overexpression of wild-type DTX1 also blocked $\mathrm{Su}(\mathrm{H})$ signaling, although the reverse was not the case. This suggests that differences in the expression levels of Notch1 coregulators, in particular DTX1, may be critical for determining the outcomes of Notch1 activation. That is, $\mathrm{Su}(\mathrm{H})$ mediated events could be blocked in cells expressing high levels of DTX1.

Whereas we found that Notch1-induced erbB2 expression in cerebellar astroglia occurs via DTX1, it has been reported that Notch1-induced erbB2 expression in cultures of cortical radial glia is blocked by a DN-Su(H) construct (Schmid et al., 2003). These results suggest that the ways in which Notch1 signaling regulates radial glia formation may differ between regions of the CNS. This difference between cortical and cerebellar radial glia could reflect distinct temporal and spatial expression levels of DTX1 and $\mathrm{Su}(\mathrm{H})$. Additional studies examining the pattern and levels of expression of DTX1 and $\mathrm{Su}(\mathrm{H})$ should provide more insight into how these pathways function throughout development.

It has been speculated that DTX-dependent Notch1 signaling may be driven by ligands that are different from those that activate $\mathrm{Su}(\mathrm{H})$ events (Kadesch, 2004). However, we have shown that Jagged1, a Notch1 ligand expressed by granule cells in the developing cerebellum, induces the expression of both BLBP and erbB2 in astroglia (Patten et al., 2003), indicating that it is capable of inducing both $\mathrm{Su}(\mathrm{H})$ - and DTX-mediated events and could be the Notch1 ligand responsible for regulating these aspects of Bergmann glia function. Nevertheless, the possibility that in vivo DTX signaling may be activated by alternate ligands should not be overlooked (Hu et al., 2003; Cui et al., 2004; Eiraku et al., 2005). One molecule we have considered is contactin, a neural cell adhesion molecule reported to activate Notch1-DTX1 signaling in oligodendrocytes (Hu et al., 2003). Granule neurons express contactin, and contactin knock-out mice have abnormal cerebellar development and are ataxic (Berglund et al., 1999), making this molecule a strong candidate for a Notch1 ligand that regulates Bergmann glia differentiation. However, we tested the effects of contactin on cerebellar astroglia and found it does not activate Notch1 signaling in cerebellar astroglia, alter glia morphology, or activate the BLBP and the erbB2 promoters (supplemental Fig. 1, available at www.jneurosci.org as supplemental material). Therefore, contactin is unlikely to play a role in the morphological differentiation of radial glia. It remains to be tested whether this molecule plays a role earlier in development in cell fate choices that drive precursor cells to become radial glia. Another molecule that has been implicated recently in Notch1 signaling in the cerebellum is Delta/Notch-like epidermal growth factor-related receptor (DNER). Eiraku et al. (2005) proposed that this putative Notch1 ligand, which is expressed by Purkinje cells, regulates Bergmann glia radial process formation through the activation of DTX1 signaling. However, because these conclusions were based on the use of DN-DTX1, which also blocks $\mathrm{Su}(\mathrm{H})$ function, it remains to be determined whether elimination of DTX1 is sufficient to eliminate the biological effects of DNERmediated Notch1 signaling.

Similar to what we observed in cerebellar astroglia, the DTX family members appear to act as positive regulators of Notch1 signaling in other cell types. For example, activation of the Notch1 receptor in myoblasts regulates their differentiation through pathways that are both dependent and independent of $\mathrm{Su}(\mathrm{H})$ (Shawber et al., 1996; Nofziger et al., 1999), the $\mathrm{Su}(\mathrm{H})$ independent pathway being positively mediated by DTX signaling (Kishi et al., 2001). Similarly, DTX1 has been shown to act as a positive regulator of Notch1 in Xenopus embryos, in which misexpression of DTX1 results in expansion of the neuroepithelium in a way similar to that induced by misexpression of activated Notch1 (Kishi et al., 2001). DTX1 also mimics Notch1 activation in a neural precursor cell line (MSN-70), in which it inhibits their differentiation, a process that is independent of $\mathrm{Su}(\mathrm{H})$ (Yamamoto et al., 2001). Interestingly, in contrast to our glia experiments in which overexpression of DTX1 has no effect on erbB2 expression and radial glia morphology, in some of these studies (Kishi et al., 2001; Yamamoto et al., 2001), overexpression of DTX1 alone was sufficient to mimic Notch1 activation, in some cases even activating processes previously considered to depend on $\mathrm{Su}(\mathrm{H})$ (Hori et al., 2004). Therefore, the effects of DTX in different cell types can vary, but the reasons for these differences remain to be determined.

Some reports showed that DTX1 can also negatively regulate Notch1 signaling. Sestan et al. (1999) demonstrated that Notch1 activation inhibits neurite outgrowth in embryonic day 15-16 cortical neurons and that overexpression of DTX1 relieves this inhibition. However, based on our results, it is remains to be determined whether these observations reflected DTX1-induced transcriptional activation of genes important for neurite outgrowth or whether they resulted from DTX1 overexpression blocking a $\mathrm{Su}(\mathrm{H})$-dependent inhibition of process outgrowth. DTX1 also has ubiquitin ligase activity (Takeyama et al., 2003; Liu and Lai, 2005), which has lead to the speculation that it could act as a negative regulator of Notch1 by mediating its ubiquitination and degradation (Kanwar and Fortini, 2004) or as a positive regulator by promoting internalization and stabilization of NICD (Hori et al., 2004). However, because in our experiments DNDTX1, which lacks the ubiquitin ligase domain (Takeyama et al., 2003), inhibits Notch1-induced erbB expression and radial glia formation, it is unlikely that the negative effects of DN-DTX1 were mediated by ubiquitination of the Notch1 receptor.

In summary, we examined how activation of the Notch1 receptor leads to multiple downstream transcriptional events, involving $\mathrm{Su}(\mathrm{H})$ and DTX1, that are critical for the formation of radial glia. It will be interesting to explore further the biological significance of the interactions between the DTX1 and $\mathrm{Su}(\mathrm{H})$ pathways suggested by our experiments in glia and to test whether they are relevant for other cell types that are regulated by Notch1 (Nofziger et al., 1999; Sestan et al., 1999; Kishi et al., 2001). Importantly, unlike Su(H), DTX1 has no known DNA binding domains. Identification of DNA-binding proteins that form part of the Notch1-DTX1 complex could further elucidate how this molecule mediates Notch1 effects on transcription.

\section{References}

Anthony TE, Mason HA, Gridley T, Fishell G, Heintz N (2005) Brain lipidbinding protein is a direct target of Notch signaling in radial glial cells. Genes Dev 19:1028-1033.

Berglund EO, Murai KK, Fredette B, Sekerkova G, Marturano B, Weber L, Mugnaini E, Ranscht B (1999) Ataxia and abnormal cerebellar microorganization in mice with ablated contactin gene expression. Neuron 24:739-750.

Borghesani PR, Peyrin JM, Klein R, Rubin J, Carter AR, Schwartz PM, Luster A, Corfas G, Segal RA (2002) BDNF stimulates migration of cerebellar granule cells. Development 129:1435-1442. 
Cui XY, Hu QD, Tekaya M, Shimoda Y, Ang BT, Nie DY, Sun L, Hu WP, Karsak M, Duka T, Takeda Y, Ou LY, Dawe GS, Yu FG, Ahmed S, Jin LH, Schachner M, Watanabe K, Arsenijevic Y, Xiao ZC (2004) NB-3/Notch1 pathway via Deltex1 promotes neural progenitor cell differentiation into oligodendrocytes. J Biol Chem 279:25858-25865.

Eiraku M, Tohgo A, Ono K, Kaneko M, Fujishima K, Hirano T, Kengaku M (2005) DNER acts as a neuron-specific Notch ligand during Bergmann glial development. Nat Neurosci 8:873-880.

Feng L, Heintz N (1995) Differentiating neurons activate transcription of the brain lipid-binding protein gene in radial glia through a novel regulatory element. Development 121:1719-1730.

Feng L, Hatten ME, Heintz N (1994) Brain lipid-binding protein (BLBP): a novel signaling system in the developing mammalian CNS. Neuron 12:895-908.

Goldowitz D, Hamre K (1998) The cells and molecules that make a cerebellum. Trends Neurosci 21:375-382.

Hatten ME (1985) Neuronal regulation of astroglial morphology and proliferation in vitro. J Cell Biol 100:384-396.

Hori K, Fostier M, Ito M, Fuwa TJ, Go MJ, Okano H, Baron M, Matsuno K (2004) Drosophila deltex mediates suppressor of Hairless-independent and late-endosomal activation of Notch signaling. Development 131:5527-5537.

Hsieh JJ, Henkel T, Salmon P, Robey E, Peterson MG, Hayward SD (1996) Truncated mammalian Notch1 activates CBF1/RBPJk-repressed genes by a mechanism resembling that of Epstein-Barr virus EBNA2. Mol Cell Biol 16:952-959.

Hu QD, Ang BT, Karsak M, Hu WP, Cui XY, Duka T, Takeda Y, Chia W, Sankar N, Ng YK, Ling EA, Maciag T, Small D, Trifonova R, Kopan R, Okano H, Nakafuku M, Chiba S, Hirai H, Aster JC, et al. (2003) F3/ contactin acts as a functional ligand for Notch during oligodendrocyte maturation. Cell 115:163-175.

Irvin DK, Zurcher SD, Nguyen T, Weinmaster G, Kornblum HI (2001) Expression patterns of Notch1, Notch2, and Notch3 suggest multiple functional roles for the Notch-DSL signaling system during brain development. J Comp Neurol 436:167-181.

Kadesch T (2004) Notch signaling: the demise of elegant simplicity. Curr Opin Genet Dev 14:506-512.

Kanwar R, Fortini ME (2004) Notch signaling: a different sort makes the cut. Curr Biol 14:R1043-R1045.

Kishi N, Tang Z, Maeda Y, Hirai A, Mo R, Ito M, Suzuki S, Nakao K, Kinoshita T, Kadesch T, Hui C, Artavanis-Tsakonas S, Okano H, Matsuno K (2001) Murine homologs of deltex define a novel gene family involved in vertebrate Notch signaling and neurogenesis. Int J Dev Neurosci 19:21-35.

Lai EC (2002) Keeping a good pathway down: transcriptional repression of Notch pathway target genes by CSL proteins. EMBO Rep 3:840-845.

Liu WH, Lai MZ (2005) Deltex regulates T-cell activation by targeted degradation of active MEKK1. Mol Cell Biol 25:1367-1378.

Mason CA, Edmondson JC, Hatten ME (1988) The extending astroglial process: development of glial cell shape, the growing tip, and interactions with neurons. J Neurosci 8:3124-3134.

Matsuno K, Diederich RJ, Go MJ, Blaumueller CM, Artavanis-Tsakonas S (1995) Deltex acts as a positive regulator of Notch signaling through interactions with the Notch ankyrin repeats. Development 121:2633-2644.

Matsuno K, Eastman D, Mitsiades T, Quinn AM, Carcanciu ML, Ordentlich P, Kadesch T, Artavanis-Tsakonas S (1998) Human deltex is a conserved regulator of Notch signalling. Nat Genet 19:74-78.

Messersmith DJ, Murtie JC, Le TQ, Frost EE, Armstrong RC (2000) Fibroblast growth factor 2 (FGF2) and FGF receptor expression in an experimental demyelinating disease with extensive remyelination. J Neurosci Res 62:241-256.

Nofziger D, Miyamoto A, Lyons KM, Weinmaster G (1999) Notch signaling imposes two distinct blocks in the differentiation of $\mathrm{C} 2 \mathrm{C} 12$ myoblasts. Development 126:1689-1702.

Patten BA, Peyrin JM, Weinmaster G, Corfas G (2003) Sequential signaling through Notch1 and erbB receptors mediates radial glia differentiation. J Neurosci 23:6132-6140.

Peles E, Nativ M, Campbell PL, Sakurai T, Martinez R, Lev S, Clary DO Schilling J, Barnea G, Plowman GD, Grumet M, Schlessinger J (1995) The carbonic anhydrase domain of receptor tyrosine phosphatase beta is a functional ligand for the axonal cell recognition molecule contactin. Cell $82: 251-260$.

Rakic P (1971a) Guidance of neurons migrating to the fetal monkey neocortex. Brain Res 33:471-476.

Rakic P (1971b) Neuron-glia relationship during granule cell migration in developing cerebellar cortex. A Golgi and electron microscopic study in Macacus rhesus. J Comp Neurol 141:283-312.

Ramain P, Khechumian K, Seugnet L, Arbogast N, Ackermann C, Heitzler P (2001) Novel Notch alleles reveal a Deltex-dependent pathway repressing neural fate. Curr Biol 11:1729-1738.

Rio C, Rieff HI, Qi P, Khurana TS, Corfas G (1997) Neuregulin and erbB receptors play a critical role in neuronal migration. Neuron 19:39-50.

Rubinson DA, Dillon CP, Kwiatkowski AV, Sievers C, Yang L, Kopinja J, Rooney DL, Ihrig MM, McManus MT, Gertler FB, Scott ML, Van Parijs L (2003) A lentivirus-based system to functionally silence genes in primary mammalian cells, stem cells and transgenic mice by RNA interference. Nat Genet 33:401-406.

Schmid RS, McGrath B, Berechid BE, Boyles B, Marchionni M, Sestan N, Anton ES (2003) Neuregulin 1-erbB2 signaling is required for the establishment of radial glia and their transformation into astrocytes in cerebral cortex. Proc Natl Acad Sci USA 100:4251-4256.

Sestan N, Artavanis-Tsakonas S, Rakic P (1999) Contact-dependent inhibition of cortical neurite growth mediated by notch signaling. Science 286:741-746.

Shawber C, Nofziger D, Hsieh JJ, Lindsell C, Bogler O, Hayward D, Weinmaster G (1996) Notch signaling inhibits muscle cell differentiation through a CBF1-independent pathway. Development 122:3765-3773.

Sotelo C, Changeux JP (1974) Bergmann fibers and granular cell migration in the cerebellum of homozygous weaver mutant mouse. Brain Res 77:484-491.

Storck S, Delbos F, Stadler N, Thirion-Delalande C, Bernex F, Verthuy C, Ferrier P, Weill JC, Reynaud CA (2005) Normal immune system development in mice lacking the Deltex-1 RING finger domain. Mol Cell Biol 25:1437-1445.

Takeyama K, Aguiar RC, Gu L, He C, Freeman GJ, Kutok JL, Aster JC, Shipp MA (2003) The BAL-binding protein BBAP and related Deltex family members exhibit ubiquitin-protein isopeptide ligase activity. J Biol Chem 278:21930-21937.

Wettstein DA, Turner DL, Kintner C (1997) The Xenopus homolog of Drosophila suppressor of Hairless mediates Notch signaling during primary neurogenesis. Development 124:693-702.

White MR, Hung MC (1992) Cloning and characterization of the mouse neu promoter. Oncogene 7:677-683.

Xu T, Artavanis-Tsakonas S (1990) deltex, a locus interacting with the neurogenic genes, Notch, Delta and mastermind in Drosophila melanogaster. Genetics 126:665-677.

Yamamoto N, Yamamoto Si S, Inagaki F, Kawaichi M, Fukamizu A, Kishi N, Matsuno K, Nakamura K, Weinmaster G, Okano H, Nakafuku M (2001) Role of Deltex-1 as a transcriptional regulator downstream of the Notch receptor. J Biol Chem 276:45031-45040.

Yue Q, Groszer M, Gil JS, Berk AJ, Messing A, Wu H, Liu X (2005) PTEN deletion in Bergmann glia leads to premature differentiation and affects laminar organization. Development 132:3281-3291. 